\section{Unusual presentation of rate-dependent intermittent transient bundle branch block in a patient with head injury}

\author{
Gaurav Singh Tomar ${ }^{1}$, Shailendra Kumar', \\ Anudeep Saxena ${ }^{1,2}$, Surya Kumar Dube', \\ Keshav Goyal ${ }^{1}$
}

Rate-dependent left bundle branch block (LBBB) in neurosurgical intensive care unit (NSICU) is a rare entity. We report an unusual presentation of heart rate-dependent transient, but intermittent LBBB in a patient with traumatic brain injury (TBI) without previous history of cardiac disease.

A 41-year-old male patient without co-morbidities, suffering with right fronto-temporo-parietal subdural haematoma (SDH) of 90cc approximately following head injury presented with Glasgow coma score (GCS) of $4 / 15$ (motor response 2 ) in the emergency department. He was operated on the same day. In the NSICU, on day 2 , it was noticed that the patient's electrocardiogram showed changes of LBBB or tachy-brady syndrome intermittently. This coincided with increase in patient's heart rate, as evident on the 12-lead ECG [Figure 1]. An acute myocardial event was suspected. This patient was evaluated by cardiologist and intensivist. Serial Troponin-T/I and bedside 2D-echocardiography was performed, which was within normal limits. Intravenous metoprolol dose $50 \mathrm{mg}$ was administered for the high

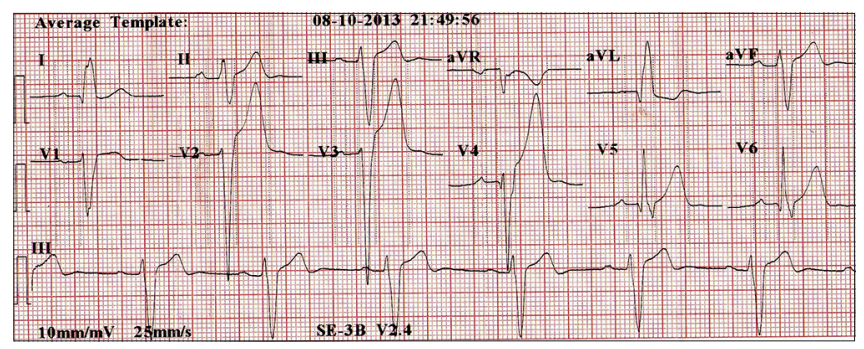

Figure 1: Electrocardiogram showing rate-dependent transient left bundle branch block in a young patient with traumatic brain injury blood pressure and heart rate but had to be discontinued soon after the LBBB pattern reverted to normal and the patient suffered sinus bradycardia. Undesirable sympathetic stimulation leading to LBBB may occur at the time of tracheal or oropharyngeal suctioning and during periodic change in position of patient in ICU. This was managed by deepening the plane of sedation and using sympatholytic drugs such as esmolol and lignocaine.

Isolated LBBB in a healthy young adult may be benign and of lesser significance. However, in a known hypertensive or older patients, it may signify a progressive degenerating myocardium involving cardiac conduction system, coronary artery disease (CAD) or cardiomyopathy. ${ }^{[1,2]}$ The rate-dependent bundle branch block is defined as an intraventricular conduction defect that may return to sinus rhythm at lower heart rates. ${ }^{[3]}$ It is important to identify the rate-dependent LBBB and further differentiate it from cardiac disease-associated LBBB in a critically injured patient. For the diagnosis of rate-dependent LBBB without any coexisting cardiac co-morbidity, manoeuvres like unilateral carotid massage, and pharmacological drugs like esmolol, labetolol and lignocaine (preservative-free) may be helpful. They decrease the heart rate and revert this kind of conduction block to normal sinus rhythm. We realized that in our patient, manoeuvres such as endotracheal suctioning without adequate sedation was precipitating transient blocks. Holter examination, which could have given us more information regarding the arrhythmia couldn't be conducted in our patient because of the lack of availability. In a chronic hypertensive patient with LBBB, it is preferentially better to go for further evaluation such as complete cardiac evaluation, with 2D/stress echocardiogram, in order to rule out any associated CAD. ${ }^{[4]}$

In conclusion, while managing LBBB in the absence of any co-morbidities in a critically injured patient, we should take into consideration rate-dependent LBBB as one of the differential diagnosis. We should avoid all the stimuli in routine intensive care practice that deleteriously elevates the heart rate. We suggest deepening the plane of sedation or using sympatholytic drugs to blunt this response at the time of suctioning, during periodic position change and any interventions in patients with TBI having low GCS.

${ }^{1}$ Department of Neuroanesthesiology and Critical Care, ${ }^{2}$ Jai Prakash Narayan Apex Trauma Centre, All India Institute of Medical Sciences, New Delhi, India

Address for correspondence:

Dr. Gaurav Singh Tomar, Department of Neuroanesthesiology, $6^{\text {th }}$ floor, Neuroscience Centre, All India Institute of Medical Sciences, New Delhi - 110029 ,

India. E-mail: spunkygst@gmail.com 


\section{REFERENCES}

1. Grady TA, Chiu AC, Snader CE, Marwick TH, Thomas JD, Pashkow FJ, et al.Prognosticsignificance of exercise-induced left bundle-branch block. JAMA 1998;14:279:153-6.

2. Fahy GJ, Pinski SL, Miller DP, McCabe N, Pye C, Walsh MJ, et al. Natural history of isolated bundle branch block. Am J Cardiol 1996;77:1185-90.

3. Wagner GS. Intraventricular conduction abnormalities. In: Wagner GS, editor. Marriott's Practical Electrocardiography. Philadelphia: Lipincott Williams and Wilkins; 2001. p. 96-116.

4. Mishra S, Nasa P, Goyal GN, Khurana H, Gupta D, Bhatnagar S. The rate dependent bundle branch block-transition from left bundle branch block to intraoperative normal sinus rhythm. Middle East J Anesthesiol 2009;20:295-8.

\begin{tabular}{|l|l|}
\hline \multicolumn{2}{|c|}{ Access this article online } \\
\hline Quick Response Code: & Website: \\
\hline$\square$ & www.jnaccjournal.org \\
\cline { 2 - 2 } & \\
\hline
\end{tabular}

